\title{
Clopidogrel and interaction with proton pump inhibitors: comparison between cohort and within person study designs
}

\author{
(ㄷ) (1) O OPEN ACCESS
}

\author{
Ian J Douglas lecturer in pharmacoepidemiology ${ }^{1}$, Stephen J W Evans professor of \\ pharmacoepidemiology ${ }^{1}$, Aroon D Hingorani professor of genetic epidemiology ${ }^{2}$, Anthony M Grosso \\ formulary pharmacist ${ }^{3}$, Adam Timmis consultant interventional cardiologist, professor ${ }^{4}$, Harry \\ Hemingway professor of clinical epidemiology ${ }^{2}$, Liam Smeeth professor of clinical epidemiology ${ }^{1}$
}

${ }^{1}$ Faculty of Epidemiology and Population Health, London School of Hygiene and Tropical Medicine, London WC1E 7HT, UK; ${ }^{2}$ Department of Epidemiology and Public Health, University College London, London WC1E 6BT; ${ }^{3}$ Pharmacy Department, University College London Hospital, London NW1 2PG; ${ }^{4}$ Department of Cardiology, Barts and The London Heart and Chest Centre, London E2 9JX

\begin{abstract}
Objective To measure the association between use of proton pump inhibitors and a range of harmful outcomes in patients using clopidogrel and aspirin.

Design Observational cohort study and self controlled case series.

Setting United Kingdom General Practice Research Database with linked data from the Myocardial Ischaemia National Audit Project (MINAP) and the Office for National Statistics (the cardiovascular disease research using linked bespoke studies and electronic records (CALIBER) collaboration)

Population 24471 patients receiving clopidogrel and aspirin.

Main outcome measures The primary outcome was death or incident myocardial infarction. Secondary outcomes were death, incident myocardial infarction, vascular death, and non-vascular death. Comparisons were made between proton pump inhibitor use and non-use.

Results Of the 24471 patients prescribed clopidogrel and aspirin, 12 $439(50 \%)$ were also prescribed a proton pump inhibitor at some time during the study. Death or incident myocardial infarction occurred in $1419(11 \%)$ patients while they were receiving a proton pump inhibitor compared with 1341 (8\%) who were not receiving a proton pump inhibitor. In multivariate analysis, the hazard ratio for the association between proton pump inhibitor use and death or incident myocardial infarction was 1.37 (95\% confidence interval 1.27 to 1.48 ). Comparable results were seen for secondary outcomes and with other 2C19 inhibitors and with non-2C19 inhibitors. With the self controlled case series design to remove the effect of differences between people, there was no association between proton pump inhibitor use and myocardial infarction, with a rate ratio of 0.75 ( 0.55 to 1.01$)$. Similarly, with the self controlled
\end{abstract}

case series there was no association with myocardial infarction for other 2C19 inhibitors/non-inhibitors.

Conclusion The lack of a specific association and the discrepancy between findings of the analyses between and within people suggests that the interaction between proton pump inhibitors and clopidogrel is clinically unimportant.

\section{Introduction}

Clopidogrel is an antiplatelet drug often given with low dose aspirin to patients with acute coronary syndrome or after ischaemic stroke, with the aim of preventing further vascular events. As clopidogrel and aspirin can both increase the risk of bleeding, a proton pump inhibitor is often co-prescribed to help reduce the risk of gastrointestinal bleeding. Over recent years there has been much debate about whether some or all proton pump inhibitors might reduce the effectiveness of clopidogrel because of a drug interaction at the cytochrome P450 2C19 enzyme. ${ }^{1-12}$ Clopidogrel is a prodrug that is metabolised to an active form, and this process is believed to occur primarily via cytochrome P450 2C19. Proton pump inhibitors inhibit this enzyme to varying degrees, and mechanistic studies show that combined use of clopidogrel with omeprazole or lansoprazole leads to a reduction in activity of clopidogrel as measured by platelet aggregation and associated biomarkers. This suggests that there is a potentially important pharmacokinetic interaction between these drugs when used at therapeutic doses. ${ }^{13}{ }^{14}$ The question of clinical importance, however, is whether this interaction has an impact on clinical outcomes. Evidence to date has been conflicting; some studies have observed an increased risk of vascular events in patients receiving clopidogrel and 
proton pump inhibitors, ${ }^{13-5}$ while others, including an underpowered randomised trial, found no increased risk. ${ }^{26-12}$ Observational study designs might not always account for confounding between people, and important differences between patients who are and are not prescribed proton pump inhibitors could account for the harmful effects observed.

We conducted two observational studies of differing designs to investigate this possible drug interaction and compared the findings. The first was a traditional cohort design, and the second was a self controlled case series design. The self controlled case series is a within person approach that eliminates fixed confounding between people that can affect case-control or cohort designs. ${ }^{15}$ The study was based on primary care computerised clinical records from the United Kingdom based General Practice Research Database, Office for National Statistics mortality statistics, and the Myocardial Ischaemia National Audit Project (MINAP). Newly established linkages between these data sources provide a powerful research tool ideal for examining questions of this nature.

\section{Methods}

\section{The General Practice Research Database}

The General Practice Research Database contains information from over 11 million patients registered at over 600 general practice surgeries in the UK. ${ }^{16}{ }^{17}$ Continuous information is recorded for each patient, including a record of each consultation, any diagnoses made, all prescribed medicines, and basic demographic data. The geographical distribution and size of practices on the database is largely representative of the population of England and Wales, and the individuals registered on the database are representative of the whole UK population in terms of age and sex. ${ }^{18}$ The quality of data held in the database is subject to rigorous checks and regular audits, and it has been successfully used to conduct over 600 peer reviewed published studies. The information obtained from the database is entirely anonymised.

\section{Myocardial Ischaemia National Audit Project database}

The Myocardial Ischaemia National Audit Project was established in 1999 to track the quality of management of heart attack in all 230 acute hospital trusts in England and Wales. ${ }^{19}$ Initially intended to capture information about ST elevation myocardial infarctions only, the audit soon expanded to include full information on all patients admitted with any suspected acute coronary syndrome. The audit is managed by the National Institute for Clinical Outcomes Research at University College London in conjunction with the British Cardiac Society. For this study, complete data on all incident myocardial infarctions recorded in the project were available from 1 January 2003 to 31 July 2009.

As part of the CALIBER (cardiovascular disease research using linked bespoke studies and electronic records) collaboration a linkage has been established between the General Practice Research Database and the Myocardial Ischaemia National Audit Project and between the General Practice Research Database and mortality records held by the Office for National Statistics. All deaths identified in England and Wales are notified to the Office for National Statistics along with details of the reported cause(s). Over 200 practices in the General Practice Research Database are fully linked to both the Myocardial Ischaemia National Audit Project and Office for National Statistics mortality data. For all patients within these practices, research data are available comprising the full General Practice
Research Database record for all patients and linked data for all patients with a valid record within Myocardial Ischaemia National Audit Project or Office for National Statistics mortality statistics. The subset of linked General Practice Research Database practices is broadly similar in profile to the full complement of practices, suggesting generalisability is maintained when the linked practices alone are used. The benefit of using these linked data is that the researcher can access a rich source of well characterised incident vascular and mortality information as well as detailed primary care data, and the linkages have already been shown to provide a useful combination of high quality data for research. ${ }^{20}{ }^{21}$

\section{Study population}

Patients were selected from all practices in the General Practice Research Database with a linkage to Myocardial Ischaemia National Audit Project and Office for National Statistics mortality statistics. Patients had to be active within the General Practice Research Database from 1 January 2003 onwards and with at least 12 months between first registration and first recorded prescription for clopidogrel. Patients also had to be concurrently prescribed aspirin as recorded in the database because aspirin can also be obtained without prescription. The overall antiplatelet benefit being experienced by patients not receiving prescribed aspirin is therefore unknown, and for this reason such patients were ineligible.

\section{Cohort analysis}

Patients enter the cohort at the latest of 1 January 2003 or first recorded prescription for clopidogrel in combination with aspirin. Follow-up was censored at the earliest of stopping treatment with clopidogrel, stopping treatment with aspirin, occurrence of an outcome of interest, death, transfer out of the practice, last data collection date for the practice, or 31 July 2009. Follow-up time was then classified according to whether the patient was receiving a proton pump inhibitor as recorded in the database, with time updated treatment status, allowing patients to fluctuate between exposed and unexposed depending on prescription patterns.

Treatment with aspirin was assumed to be continuous unless a gastrointestinal bleed was recorded, from which time treatment was assumed to be discontinued. For clopidogrel and proton pump inhibitor exposure, where possible we calculated the anticipated length of each prescription based on pack size and dosing instructions. From all prescription records where this information was available, we calculated the median length of exposure from a single prescription. This median duration was imputed for all individual prescriptions where duration was not recorded. Courses of treatment were assumed to be continuous when any apparent treatment break was less than 180 days, to allow for partial non-compliance and situations where patients might have built up treatment stocks or obtained drugs through alternative sources. The primary exposure of interest was any proton pump inhibitor. Given the varied capacity for 2C19 inhibition among individual proton pump inhibitors, however, we also restricted exposure to strong inhibitors only (omeprazole, lansoprazole, and esomeprazole). We examined other drugs likely to be used over long periods by patients taking clopidogrel as secondary exposures; other strong 2C19 inhibitors included fluoxetine/paroxetine. Citalopram and ranitidine were selected as a weak/non-2C19 inhibitors, with ranitidine used for similar indications to proton pump inhibitors. ${ }^{22}$ 


\section{Outcomes}

The primary outcome was a composite end point of all cause mortality or incident myocardial infarction. Mortality was determined from Office for National Statistics mortality records, with incident myocardial infarction determined by Myocardial Ischaemia National Audit Project records. Secondary end points included incident myocardial infarction, all cause mortality, and mortality attributed to vascular and non-vascular causes, determined with cause of death information recorded by the Office for National Statistics. Vascular causes of death were all those assigned an ICD-10 (international classification of diseases, 10th revision) code for "diseases of the circulatory system" as the underlying cause.

\section{Modelling strategy}

Cox regression was carried out with Stata 11 (StataCorp, College Station, TX, USA). Adjustments were made for age and sex, with further adjustments considered for the following covariates obtained from General Practice Research Database patient records; smoking status, alcohol status, body mass index (BMI) categorised as $<20,20-25$ or $>25$, diabetes, coronary heart disease, peripheral vascular disease, ischaemic stroke, and cancer. Patient status for each covariate was updated as relevant at any change in exposure to a proton pump inhibitor. For the small proportion of patients with missing status for smoking, BMI, and alcohol intake, "missing" was included as an extra category. Multiple imputation was considered, but these data were not considered to be missing at random. Each covariate was added to the model and retained on the basis of likelihood ratio testing $(\mathrm{P}<0.05)$

\section{Self controlled case series analysis}

The self controlled case series design method is derived from rate modelling with a Poisson distribution and is comparable with cohort methodology. It relies on comparisons within people in a population of individuals who have both the outcome and exposure of interest. ${ }^{15}$ Incidence rate ratios are derived, comparing the rate of events during exposed periods of time with the rate during all other observed time periods. A major advantage of this design is that the potential confounding effect of both recorded and unrecorded characteristics that vary between individuals, but are fixed over time within individuals, is removed. Age, which varies over time, is allowed for in the analysis.

For each participant within the cohort we identified all of those with at least one incident myocardial infarction during their follow-up time as defined above. Periods of follow-up with no exposure to a proton pump inhibitor are classified as baseline, and follow-up time was further subdivided by age in single year bands. For patients included in the self controlled case series analysis, follow-up is not censored at the occurrence of the event, as later exposed and unexposed periods of time need to be included in the analysis. Figure 1 illustrates a typical timeline $\Downarrow$. As the self controlled case series design is well suited to the study of acute and recurrent events, we used it to examine the effect of proton pump inhibitor exposure on the occurrence of myocardial infarction. Patients who had a myocardial infarction but were never exposed to a proton pump inhibitor were also included in the analysis to improve the adjustment for age. Similarly, we also included those who were exposed to a proton pump inhibitor for the entire observation period.

Other investigators using the self controlled case series design have found that during a short period of time before the exposure of interest the risk of the event of interest might be substantially different from other baseline time periods. ${ }^{23}$ For this reason we chose to separately analyse the two week period before use of a proton pump inhibitor (see fig 1).

\section{Model checking and sensitivity analyses}

We investigated the proportional hazards assumption underpinning Cox regression for the cohort analysis across age categories. As a sensitivity analysis we examined exposure to strong 2C19 inhibitors only (omeprazole, esomeprazole, and lansoprazole). Because of missing data for smoking, BMI, and alcohol consumption, we performed a complete case analysis for comparison. For the self controlled case series analysis we repeated all analyses without including a separate two week lead in period and assumed all unexposed time was part of the baseline risk period. The purpose of the lead in period in the primary analysis is to allow for the possibility that the outcome of interest could lead to a short term change (often a decrease) in the chance of being prescribed the exposure of interest. This would effectively reduce the incidence of events during the lead in period, leading to a slight underestimate of the risk in the baseline period. We also varied the period of time over which continued drug exposure was assumed, alternatively allowing periods of 90 or 60 days. We carried out an analysis restricted to the first myocardial infarction during observation to check the assumption of event independence. To account for possible bias from event dependent observation censoring we performed an analysis restricted to patients who were still alive three months after their first myocardial infarction during the observation period. Finally, to check whether the occurrence of a myocardial infarction can lead to a subsequent change in the risk of receiving a proton pump inhibitor, we restricted our analysis to patients who had a first myocardial infarction before the observation period began.

\section{Results}

We identified 24471 patients receiving clopidogrel in combination with aspirin and with at least 12 months' registration in the General Practice Research Database before their first prescription for clopidogrel. Table 1 shows the background details for these patients $\Downarrow$. Of these, 9111 (37\%) also received a proton pump inhibitor from the date of first clopidogrel prescription. Those receiving proton pump inhibitors were on average two years older than those who did not use a proton pump inhibitor, with a median age of 71 years. Fifty eight percent of users were men, compared with $65 \%$ of non-users. Users of a proton pump inhibitor were also more likely to have diabetes ( $34 \% v 29 \%)$, peripheral vascular disease $(12 \% \vee 11 \%)$, or cancer (15\% v 13\%) (table 1).

During follow-up, use of proton pump inhibitor was sporadic in some patients and started later on in others. In total, 12439 $(50 \%)$ patients received a proton pump inhibitor at some point during the study period. Conversely, 16900 (69\%) patients had at least some follow-up time with no exposure to a proton pump inhibitor.

\section{Cohort analysis Primary exposure (any proton pump inhibitor)}

During follow-up (median 303 days), 2760 (11\%) patients had the primary outcome of all cause mortality or incident myocardial infarction. In total $1419(11 \%)$ events occurred during periods of exposure to a proton pump inhibitor, while $1341(8 \%)$ occurred during periods of no exposure (table $2 \Downarrow$ ). The crude hazard ratio for this association was $1.41(95 \%$ 
confidence interval 1.31 to 1.52). After adjustment for age, sex, BMI, smoking, alcohol, diabetes, peripheral vascular disease, coronary heart disease, ischaemic stroke, and cancer the hazard ratio was 1.37 (1.27 to 1.48 ; fig $2 \Downarrow$ ).

In total, 2228 (9\%) patients died during the study period; 1170 (9\%) were exposed to a proton pump inhibitor at the time, compared with 1058 (6\%) not exposed to a proton pump inhibitor. The crude hazard ratio for the association between exposure and all cause mortality was 1.44 (1.32 to 1.56$)$, with a fully adjusted figure of 1.40 (1.29 to 1.52 ; fig 2 ). When we considered mortality from underlying vascular causes only, 1226 patients died, with $612(5 \%)$ exposed to a proton pump inhibitor and 614 (4\%) unexposed. The crude hazard ratio for this association was 1.29 (1.15 to 1.44$)$, with a fully adjusted figure of 1.25 (1.12 to 1.40; fig 2). For mortality from underlying causes other than vascular, 1002 patients died during follow-up; $558(4 \%)$ while exposed to a proton pump inhibitor and 444 (3\%) while unexposed. This association gave a crude hazard ratio of 1.64 (1.44 to 1.85) and a fully adjusted figure of 1.61 (1.42 to 1.82 ; fig 2 ).

In the secondary analysis, in which we restricted the definition of proton pump inhibitor to only those known to be strong inhibitors of CYP450 2C19, the results obtained were similar (table 3).

\section{Secondary exposures (paroxetine/fluoxetine, citalopram, ranitidine)}

The non-2C19 inhibiting $\mathrm{H} 2$ receptor antagonist ranitidine was used by 1561 patients at some point during follow-up, 105 (7\%) of whom had the primary outcome of all cause mortality or incident myocardial infarction while receiving ranitidine (table $4 \Downarrow)$. This compared with $2655(11 \%)$ patients with the primary outcome during unexposed periods of follow-up, giving an adjusted hazard ratio of 1.20 (0.99 to 1.46). The adjusted hazard ratios for other outcomes were 1.25 (1.01 to 1.55$)$ for all cause mortality, 1.07 (0.72 to 1.59 ) for incident myocardial infarction, 1.06 (0.78 to 1.45 ) for vascular mortality, and 1.49 (1.11 to 1.99) for non-vascular mortality (see fig 2).

When we split follow-up time according to paroxetine or fluoxetine exposure (strong 2C19 inhibiting antidepressants), 1143 patients were exposed to either antidepressant at some point during follow-up (table $5 \Downarrow$ ). In total, 109 patients had the primary outcome during paroxetine/fluoxetine exposure time (10\%), compared with 2651 during unexposed time (11\%). The fully adjusted hazard ratio for this association was 1.42 (1.17 to 1.72). For other outcomes of interest the fully adjusted hazard ratios were 1.49 (1.20 to 1.84$)$ for all cause mortality, $1.13(0.75$ to 1.69 ) for incident myocardial infarction, 1.29 (0.95 to 1.75$)$ for vascular mortality, and 1.73 (1.29 to 2.31) for non-vascular mortality (fig 2).

By contrast, the weak/non-2C19 inhibiting antidepressant citalopram was used by 1930 patients during follow-up, and $207(11 \%)$ of these had the primary outcome (table $6 \Downarrow$ ). This compared with $2553(7 \%)$ in non-citalopram users and gave a fully adjusted hazard ratio of 1.52 (1.32 to 1.76). For secondary outcomes the fully adjusted hazard ratios were 1.61 (1.38 to 1.88 ) for all cause mortality, 1.14 (0.84 to 1.56$)$ for incident myocardial infarction, 1.44 (1.16 to 1.79) for vascular mortality, and 1.84 (1.48 to 2.29) for non-vascular mortality (fig 2).

\section{Self controlled case series analysis}

Among the main cohort of 2471 patients, 444 who had an incident myocardial infarction during follow-up had been exposed to a proton pump inhibitor at some point concurrently with clopidogrel and aspirin (table $7 \Downarrow$ ). Comparisons within individuals adjusted for age gave an incident rate ratio of 0.75 ( 0.55 to 1.01$)$. The result was similar when exposure was restricted to strong 2C19 inhibiting proton pump inhibitors only (table $7 \Downarrow$ ). For the secondary exposures of interest, the incident rate ratio for myocardial infarction was 0.57 (0.31 to 1.06) for ranitidine, 1.65 (0.87 to 3.15$)$ for paroxetine/fluoxetine, and 0.84 ( 0.49 to 1.45 ) for citalopram (fig 2 ). There was limited evidence of a slight increase in the risk of myocardial infarction in the two week period before exposures of interest began (incident rate ratio 1.39 ( 0.71 to 2.70 ) for periods before exposure to proton pump inhibitors), although this failed to reach significance (table $7 \Downarrow$ ). A sensitivity analysis categorising this period as baseline gave similar results to those presented.

\section{Model checking and sensitivity analyses}

There was limited evidence that the proportional hazards assumption might not have been valid for the association between proton pump inhibitor exposure and mortality outcomes (Wald test, $\mathrm{P}=0.05$ ). The hazard ratios obtained across stratified age groups, however, were not substantially different from the overall hazard ratio presented. The results of the cohort study were unchanged when we performed a complete case analysis. For the self controlled case series, the sensitivity analyses to check the assumptions underpinning the method did not differ materially from the main analysis, indicating the assumptions were likely to be valid.

\section{Discussion}

Using a traditional cohort analysis we observed an increased risk of myocardial infarction or all cause mortality among clopidogrel and aspirin users exposed to a proton pump inhibitor, with a hazard ratio of 1.37 (1.27 to 1.48$)$. We also saw a similar increased risk for the individual outcomes of all cause mortality, incident myocardial infarction, and vascular mortality.

Comparable results were seen when we looked at non-vascular mortality and drug exposures other than proton pump inhibitors, including drugs that would not be expected to interact with clopidogrel (such as ranitidine and citalopram). These observed associations with exposures and outcomes that would not be expected from the theoretical drug interaction suggest these results could be driven by important differences between proton pump inhibitor users and non-users rather than showing a causal association. When we used a within person study design to remove the influence of differences between people we found no association between proton pump inhibitor exposure and myocardial infarction, with a rate ratio of 0.75 (0.55 to 1.01$)$ in a self controlled case series.

We believe the association found in the cohort analysis is unlikely to be causal for several reasons. Firstly, the mechanism behind a possible causal association involves a pharmacokinetic drug interaction between clopidogrel and proton pump inhibitors at the CYP450 2C19 enzyme. This interaction would reduce the effectiveness of clopidogrel's antiplatelet activity and would be expected to lead specifically to an increased risk of vascular events in proton pump inhibitor users compared with non-users. While our results show such an increase in risk, the effect is not specific to vascular events, as shown by the hazard ratio for non-vascular mortality of 1.61 (1.42 to 1.82 ). One explanation for this strong and unpredicted finding could be unmeasured confounding, whereby people receiving proton pump inhibitors are at an increased risk for harmful outcomes, independent of proton pump inhibitor use. The baseline characteristics of users and non-users suggest that users are older and have more 
comorbidities than non-users, which would support this hypothesis. Although we adjusted for several confounding factors, it is likely that unadjusted confounding remains, whether from illnesses we have not been able to measure, through systematic differences in the severity of underlying diseases, which we were unable to determine or incomplete ascertainment of the known confounders.

Secondly, the results of secondary analyses examining the effects of other long term drug use are revealing. The results for other strong CYP450 2C19 inhibitors such as paroxetine/fluoxetine mirror those seen for proton pump inhibitors, which is consistent with an interaction at the $2 \mathrm{C} 19$ enzyme. The similar results seen for ranitidine and citalopram, however, would not be predicted from the pharmacokinetics of these drugs. An alternative and plausible explanation is that people who are prescribed long term drug treatment in addition to clopidogrel are inherently at higher risk of harmful outcomes but not through a causal association with the treatments they receive. Intuitively, this makes sense as "illness" is itself a risk factor for adverse outcomes and is clearly also associated with an increased chance of receiving drugs. Such confounding can be difficult to overcome in non-randomised studies of drug use.

The results of the self controlled case series analysis also suggest that the cohort results could be explained by confounding between people. The self controlled case series design removes all fixed confounding between individuals as the comparisons are made within an individual, relying on patients who have both exposed and unexposed periods for the main comparison of interest. These results provide no evidence of an increased risk of incident myocardial infarction during periods when patients are exposed to proton pump inhibitors (incident rate ratio 0.75 ( 0.55 to 1.01$)$ for proton pump inhibitor, 0.57 (0.31 to 1.06 ) for ranitidine, or 0.84 (0.49 to 1.45 ) for citalopram). The near protective effect seen with proton pump inhibitors and ranitidine is difficult to explain; it could represent higher adherence to aspirin and clopidogrel during periods of co-prescribing with these drugs, though this is not testable in the present study. Although the incident rate ratio for paroxetine/fluoxetine was raised (1.65), this estimate was based on a relatively small number of events and the $95 \%$ confidence interval of 0.87 to 3.15 suggests it could well be a chance finding.

Taken together, a plausible explanation for our results is that the increased risk of both vascular and non-vascular harmful outcomes seen in patients receiving proton pump inhibitors and other long term drugs could be caused by confounding between people. Although accounting for such confounding can be difficult, the use of approaches such as the self controlled case series, which is less prone to differences between people, can solve this problem. The lack of association seen between proton pump inhibitor use and myocardial infarction with this approach argues against a clinically relevant interaction between clopidogrel and proton pump inhibitors.

\section{Strengths and limitations of study}

The main advantages of this study are the large sample size taken from a well validated population based data source and the use of newly established data linkages allowing accurate ascertainment of incident myocardial infarction cases and recorded causes of death.

Some limitations could arise from the possibility of imperfect recording of exposures and outcomes. The Myocardial Ischaemia National Audit Project does not record data for patients who die before they reach hospital, and so these cases are systematically missing in our ascertainment of incident myocardial infarction. It is unlikely that underascertainment would happen differently for proton pump inhibitor users and non-users and would therefore be expected to bias any result towards the null. It is also unlikely that this would have any impact on the primary outcome, as all cause mortality includes patients who die before arriving at hospital.

Exposure status was probably incorrectly assigned to some extent as it was determined from prescribing records and we cannot know whether patients obtained all drugs from the pharmacist or took them as directed. For our analysis we had to make an assumption about likely exposure status for all drugs during apparent gaps in prescribing records. We chose to consider gaps of less than 180 days to indicate continued treatment, allowing for possible stockpiling or obtaining drugs from alternative sources. We also repeated analyses when the continuous treatment period was assumed to be shorter (60 or 90 days), and these alternative assumptions had no substantial effect on the results presented here.

\section{Comparison with other studies}

These results need to be viewed in the context of other studies examining this drug interaction. Our overall findings are consistent with several studies that also failed to find an association between proton pump inhibitors and less favourable outcomes in clopidogrel users. ${ }^{2}{ }^{6-12}$ Of note, the case control study by Valkhoff et $\mathrm{al}^{12}$ did find a harmful association but when comparisons were restricted to former or current users of proton pump inhibitors the association was not found, again suggesting a biased result driven by confounding between people. The only randomised trial designed to specifically answer this question was unfortunately stopped early and so is underpowered and unable to rule out a clinically relevant effect. ${ }^{11}$ The available results, however, did not point towards any harmful association.

Of note, the 2C19 enzyme is subject to genotypic variation, with some people having naturally poor 2C19 metabolic activity. It might be expected that poor metabolisers would experience reduced benefit from taking clopidogrel if the $2 \mathrm{C} 19$ pathway is critical for the metabolism of clopidogrel to its active form. A recent meta-analysis, however, found that $2 \mathrm{C} 19$ genotype does not influence clopidogrel effectiveness. ${ }^{24}$ This finding questions the critical role of the $2 \mathrm{C} 19$ pathway for clopidogrel effectiveness; it is possible that other pathways compensate in patients with compromised 2C19 activity.

The findings from our cohort analysis are also consistent with several studies showing an association between proton pump inhibitor use and vascular harm, ${ }^{13-5}$ but we believe we have shown why this association is unlikely to be causal. In extreme cases the magnitude of harm observed by others was greater than the maximum likely protective effect of clopidogrel, ${ }^{45}$ again suggesting an alternative explanation is needed for the results. Nonetheless, some studies have preferentially identified a harmful effect of strong CYP450 2C19 inhibitors but not weak inhibitors, a finding that is not readily explained. ${ }^{1}$ Because of prescribing patterns in the UK, more than $90 \%$ of proton pump inhibitor use is accounted for by lansoprazole, omeprazole, and esomeprazole, all of which are strong inhibitors. When we restricted our exposure to only these proton pump inhibitors, the results were similar.

\section{Conclusions and policy implications}

In conclusion, these results suggest that the drug interaction between proton pump inhibitors and clopidogrel does not result in clinical harm. The observed associations between proton 
pump inhibitor use and several harmful outcomes are likely to be non-causal and could be explained by differences between people. An analysis within individuals, which removes the effect of differences between people, found no association between proton pump inhibitor use and myocardial infarction. Current guidelines from the American College of Cardiology Foundation/American Heart Association recognise the conflicting nature of previous studies of this question and recommend re-evaluation of the need for proton pump inhibitor use in this setting. ${ }^{25}$ The use of a proton pump inhibitor with clopidogrel and aspirin is well proved to prevent harm through gastrointestinal bleeding, and we should continue to consider proton pump inhibitors as important prophylactic drugs in patients at high risk.

The CALIBER (cardiovascular disease research using linked bespoke studies and electronic records) study is funded by the Wellcome Trust (086091/Z/08/Z, P I Hemingway) and the National Institute for Health Research (RP-PG-0407-10314, P I Hemingway). The protocol for CALIBER is registered at Clinicaltrials.gov (NCT01231867).

Contributors: IJD conceived the study, analysed the data, interpreted the results, and wrote the paper. SJWE, ADH, AMG, AT, HH, and LS contributed to the study design, interpretation of results and drafting the paper. IJD is guarantor.

Funding: IJD is funded by a Medical Research Council Methodology Fellowship and LS is funded by a Wellcome Trust Fellowship. AT acknowledges the support of Barts Cardiovascular Biomedical Research Unit funded by the National Institute for Health Research. The funders played no role in the design and conduct of the study; collection, management, analysis, and interpretation of the data; and preparation, review, or approval of the manuscript.

Competing interests: All authors have completed the ICMJE uniform disclosure form at www.icmje.org/coi_disclosure.pdf (available on request from the corresponding author) and declare: IJD consults for GlaxoSmithKline, Takeda, and Gilead, and holds stock in GlaxoSmithKline; LS consults for GlaxoSmithKline.

Ethical approval: The study was approved by the Independent Scientific Advisory Group of the General Practice Research Database, the Myocardial Ischaemia National Audit Project Academic Group, and the London School of Hygiene and Tropical Medicine ethics committee.

Data sharing: No additional data available.

1 Juurlink DN, Gomes T, Ko DT, Szmitko PE, Austin PC, Tu JV, et al. A population-based study of the drug interaction between proton pump inhibitors and clopidogrel. CMAJ 2009:180:713-8.

2 Simon T, Verstuyft C, Mary-Krause M, Quteineh L, Drouet E, Méneveau N, et al. Genetic determinants of response to clopidogrel and cardiovascular events. N Engl J Med 2009;360:363-75.

3 Ho PM, Maddox TM, Wang L, Finn SD, Jesse RL, Peterson ED, et al. Risk of adverse outcomes with concomitant use of clopidogrel and proton pump inhibitors following acute coronary syndrome. JAMA 2009;301:937-44.

4 Aubert R, Epstein RS, Teagarden JR, Xia F, Yao J, Desta Z, et al. Proton pump inhibitors effect on clopidogrel effectiveness: the Clopidogrel Medco Outcomes Study (abstract 3998). Circulation 2008:118:S815
5 Pezalla E, Day D, Pullidath I. Initial assessment of clinical impact of a drug interaction between clopidogrel and proton pump inhibitors. J Am Coll Cardiol 2008:52:1038-9.

6 Rassen JA, Choudhry NK, Avorn J, Schneeweiss S. Cardiovascular outcomes and mortality in patients using clopidogrel with proton pump inhibitors after percutaneous coronary intervention or acute coronary syndrome. Circulation 2009;120:2322-9.

7 Dunn SP, Macaulay TE, Brennan DM, Campbell CL, Charnigo RJ, Smyth SS, et al. Baseline proton pump inhibitor use is associated with increased cardiovascular events with and without the use of clopidogrel in the CREDO Trial (abstract 3999). Circulation 2008:118:S815.

8 O'Donoghue ML, Braunwald E, Antman EM, Murphy SA, Bates ER, Rozenman Y, et al. Pharmacodynamic effect and clinical efficacy of clopidogrel and prasugrel with or without a proton-pump inhibitor: an analysis of two randomised trials. Lancet 2009;374:989-97.

9 Zairis MN, Tsiaousis GZ, Patsourakos NG, Georgilas AT, Kontos CF, Adamopoulou EN, et al. The impact of treatment with omeprazole on the effectiveness of clopidogrel drug therapy during the first year after successful coronary stenting. Can $\mathrm{J}$ Cardiol 2010;26:e54-7.

10 Ray WA, Murray KT, Griffin MR, Chung CP, Smalley WE, Hall K, et al. Outcomes with concurrent use of clopidogrel and proton-pump inhibitors: a cohort study. Ann Intern Med 2010;152:337-45.

11 Bhatt DL, Cryer BL, Contant CF, Cohen M, Lanas A, Schnitzer TJ, et al. Clopidogrel with or without omeprazole in coronary artery disease. N Engl J Med 2010;363:1909-17.

12 Valkhoff VE, 't Jong GW, Van Soest EM, Kuipers EJ, Sturkenboom MC. Risk of recurrent myocardial infarction with the concomitant use of clopidogrel and proton pump inhibitors. Aliment Pharmacol Ther 2011;33:77-88.

13 Gilard M, Arnaud B, Comily JC, Le Gal G, Lacut K, Le Calvez G, et al. Influence of omeprazole on the antiplatelet action of clopidogrel associated with aspirin: the randomized double-blind OCLA (Omeprazole CLopidogrel Aspirin) study. J Am Coll Cardiol 2008;51:256-60.

14 Small DS, Farid NA, Payne CD, Weerakkody GJ, Li YG, Brandt JT, et al. Effects of the proton pump inhibitor lansoprazole on the pharmacokinetics and pharmacodynamics of prasugrel and clopidogrel. J Clin Pharmacol 2008;48:475-84.

15 Whitaker HJ, Farrington CP, Spiessens B, Musonda P. Tutorial in biostatistics: the self-controlled case series method. Stat Med 2006;25:1768-97.

16 Lawson DH, Sherman V, Hollowell J. The general practice research database. Q J Med 1998;91:445-52.

17 The Clinical Practice research Datalink Group. The general practice research database. 2012. www.gprd.com/_docs/GPRD\%20Practice\%20Patient\%20Populations\%20Jul2011. pdf .

18 Office for National Statistics. Key health statistics from general practice 1998. Office for National Statistics, 2000:149.

19 Herrett $E$, Smeeth L, Walker L, Weston C, on behalf of the MINAP academic group, the Myocardial Ischaemia National Audit Project (MINAP). Heart 2010;96:1264-7.

20 Gallagher AM, Puri S, van Staa T. Linkage of the General Practice Research Database (GPRD) with other data sources 2011. Pharmacoepi \& Drug Safety 20(S1):528.

21 Boggon R, van Staa TP, Timmis A, Hemingway H, Ray KK, Begg A, et al. Clopidogrel discontinuation after acute coronary syndromes: frequency, predictors and associations with death and myocardial infarction-a hospital registry-primary care linked cohort (MINAP-GPRD). Eur Heart J 2011: published obnline 29 August.

22 Ogu CC, Maxa JL. Drug interactions due to cytochrome P450. BUMC Proceedings 2000;13:421-3.

23 Pratt NL, Roughead EE, Ramsay E, Salter A, Ryan P. Risk of hospitalization for stroke associated with antipsychotic use in the elderly. Drugs Aging 2010;27:885-93.

24 Bauer T, Bouman HJ, van Werkum JW, Ford NF, Ten Berg JM, Taubert D. Impact of CYP2C19 variant genotypes on clinical efficacy of antiplatelet treatment with clopidogrel: systematic review and meta-analysis. BMJ 2011;343:d4588.

25 Wright RS, Anderson JL, Adams CD, Bridges CR, Casey DE Jr, Ettinger SM, et al. 2011 ACCF/AHA focused update of the guidelines for the management of patients with unstable angina/non-ST-elevation myocardial infarction (updating the 2007 guideline): a report of the American College of Cardiology Foundation/American Heart Association Task Force on Practice Guidelines developed in collaboration with the American College of Emergency Physicians, Society for Cardiovascular Angiography and Interventions, and Society of Thoracic Surgeons. J Am Coll Cardiol 2011 May 10;57:1920-59.

Accepted: 29 May 2012

\section{Cite this as: BMJ 2012;345:e4388}

This is an open-access article distributed under the terms of the Creative Common Attribution Non-commercial License, which permits use, distribution, and reproduction in any medium, provided the original work is properly cited, the use is non commercial and is otherwise in compliance with the license. See: http://creativecommons.org/licenses/by$\mathrm{nc} / 2.0 /$ and http://creativecommons.org/licenses/by-nc/2.0/legalcode. 


\section{What is already known on this topic}

Over recent years there has been a debate over whether the pharmacokinetic drug interaction between clopidogrel and proton pump inhibitors reduces the antiplatelet effects of clopidogrel and leads to an increased risk of vascular events

Studies aiming to answer this question have had conflicting findings

\section{What this study adds}

The combination of a proton pump inhibitor with clopidogrel is associated with an increased risk of both vascular and non-vascular adverse outcomes, but that this association is unlikely to be causal

The association is probably because of important differences between proton pump inhibitor users and non-users as a comparison within individuals, which removes the effect of differences between people, found no association between the use of a proton pump inhibitor and the risk of myocardial infarction

\section{Tables}

\begin{tabular}{|c|c|c|c|c|}
\hline & \multicolumn{2}{|c|}{ Clopidogrel and aspirin } & \multirow[b]{2}{*}{$P$ value } & \\
\hline & No PPI $(n=15360)$ & PPI $(n=9111)$ & & \\
\hline Median age (years) & 68 & 71 & $<0.001$ & \\
\hline Men & $10007(65)$ & $5323(58)$ & $<0.001$ & \\
\hline \multicolumn{4}{|l|}{ BMI $\left(\mathrm{kg} / \mathrm{m}^{2}\right):$} & \\
\hline$<20$ & $480(3)$ & $429(5)$ & \multirow[t]{3}{*}{0.005} & \\
\hline $20-25$ & $3987(26)$ & $2339(26)$ & & \\
\hline$>25$ & $10004(65)$ & $5809(64)$ & & \\
\hline Missing & $889(6)$ & $534(6)$ & & \\
\hline \multicolumn{4}{|l|}{ Smoking: } & \\
\hline Non-smoker & $4781(31)$ & $2780(31)$ & \multirow[t]{4}{*}{0.005} & \\
\hline Current & $2760(18)$ & $1503(16)$ & & \\
\hline Ex-smoker & $7777(51)$ & $4799(53)$ & & \\
\hline Missing & $42(0.3)$ & $29(0.3)$ & & \\
\hline \multicolumn{4}{|l|}{ Alcohol: } & \\
\hline Non-drinker & $1528(10)$ & $1080(12)$ & \multirow[t]{7}{*}{$<0.001$} & \\
\hline Ex-drinker & $938(6)$ & $687(8)$ & & \\
\hline Amount not specified & $399(3)$ & $254(3)$ & & \\
\hline$<2$ units/day & $3060(20)$ & $1908(21)$ & & \\
\hline 3-6 units/day & $7488(49)$ & $4106(45)$ & & \\
\hline$>6$ units/day & $1180(8)$ & $606(7)$ & & \\
\hline Status unknown & $767(5)$ & $470(5)$ & & \\
\hline \multicolumn{4}{|l|}{ History of: } & \\
\hline Diabetes & $4404(29)$ & $3090(34)$ & $<0.001$ & \\
\hline Peripheral vascular disease & $1629(11)$ & $1095(12)$ & 0.001 & \\
\hline Coronary heart disease & $12198(79)$ & $7292(80)$ & 0.2 & \\
\hline Ischaemic stroke & $1571(10)$ & $954(10)$ & 0.5 & \\
\hline Cancer & $2038(13)$ & $1381(15)$ & $<0.001$ & \\
\hline
\end{tabular}


Table 2| Association between exposure to proton pump inhibitor (PPI) and vascular/mortality outcomes in patients taking clopidogrel and aspirin

\begin{tabular}{|c|c|c|c|c|c|}
\hline \multirow[b]{2}{*}{ Exposure* $^{*}$} & \multirow[b]{2}{*}{ No of people } & \multirow[b]{2}{*}{ Median follow-up (days) } & \multirow{2}{*}{$\begin{array}{l}\text { No of events (all cause mortality or } \\
\text { incident myocardial infarction) }\end{array}$} & \multicolumn{2}{|c|}{ Hazard ratio $(95 \% \mathrm{Cl})$} \\
\hline & & & & Crude & Fully adjusted $\dagger$ \\
\hline \multicolumn{6}{|c|}{ Primary outcome } \\
\hline \multicolumn{6}{|c|}{ All cause mortality or incident myocardial infarction: } \\
\hline No PPI & 16680 & 355 & 1341 & - & - \\
\hline PPI & 12345 & 301 & 1419 & 1.41 (1.31 to 1.52$)$ & 1.37 (1.27 to 1.48$)$ \\
\hline \multicolumn{6}{|c|}{ Secondary outcomes } \\
\hline \multicolumn{6}{|c|}{ All cause mortality: } \\
\hline No PPI & 16900 & 361 & 1058 & - & - \\
\hline PPI & 12439 & 312 & 1170 & 1.44 (1.32 to 1.56$)$ & 1.40 (1.29 to 1.52$)$ \\
\hline \multicolumn{6}{|c|}{ Incident myocardial infarction: } \\
\hline No PPI & 16680 & 355 & 369 & - & - \\
\hline PPI & 12345 & 301 & 365 & 1.38 (1.19 to 1.59$)$ & $1.30(1.12$ to 1.50$)$ \\
\hline \multicolumn{6}{|c|}{ Vascular mortality: } \\
\hline No PPI & 16900 & 361 & 614 & - & - \\
\hline PPI & 12439 & 312 & 612 & $1.29(1.15$ to 1.44$)$ & $1.25(1.12$ to 1.40$)$ \\
\hline \multicolumn{6}{|c|}{ Non-vascular mortality: } \\
\hline No PPI & 16900 & 361 & 444 & - & - \\
\hline PPI & 12439 & 312 & 558 & 1.64 (1.44 to 1.85$)$ & 1.61 (1.42 to 1.82$)$ \\
\hline
\end{tabular}

${ }^{*}$ Captures whether patients were ever counted in this exposure group during follow-up.

†Adjusted for age, sex, BMI, smoking, alcohol, diabetes, peripheral vascular disease, coronary heart disease, ischaemic stroke, cancer.w 
Table 3| Association between exposure to strong CYP450 2C19 inhibiting proton pump inhibitor ${ }^{\star}$ and vascular/mortality in patients taking clopidogrel and aspirin

\begin{tabular}{|c|c|c|c|c|c|}
\hline \multirow[b]{2}{*}{ Exposure† } & \multirow[b]{2}{*}{ No of patients } & \multirow[b]{2}{*}{ Median follow-up (days) } & \multirow{2}{*}{$\begin{array}{l}\text { No of events (all cause mortality or } \\
\text { incident myocardial infarction) }\end{array}$} & \multicolumn{2}{|c|}{ Hazard ratio $(95 \% \mathrm{Cl})$} \\
\hline & & & & Crude & Fully adjustedł \\
\hline \multicolumn{6}{|c|}{ Primary outcome } \\
\hline \multicolumn{6}{|c|}{ All cause mortality or incident myocardial infarction: } \\
\hline No strong PPI & 17405 & 359 & 1414 & - & - \\
\hline Strong PPI & 11910 & 292 & 1346 & $1.43(1.33$ to 1.54$)$ & $1.39(1.29$ to 1.50$)$ \\
\hline \multicolumn{6}{|c|}{ Secondary outcomes } \\
\hline \multicolumn{6}{|c|}{ All cause mortality: } \\
\hline No strong PPI & 17425 & 367 & 1116 & - & - \\
\hline Strong PPI & 12012 & 299 & 1112 & $1.45(1.34$ to 1.58$)$ & $1.43(1.31$ to 1.56$)$ \\
\hline \multicolumn{6}{|c|}{ Incident myocardial infarction: } \\
\hline No strong PPI & 17405 & 359 & 391 & - & - \\
\hline Strong PPI & 11910 & 292 & 343 & $1.37(1.20$ to 1.60$)$ & $1.29(1.12$ to 1.49$)$ \\
\hline \multicolumn{6}{|c|}{ Vascular mortality: } \\
\hline No strong PPI & 17425 & 367 & 655 & - & - \\
\hline Strong PPIs & 12012 & 299 & 571 & 1.27 (1.13 to 1.42$)$ & $1.28(1.15$ to 1.44$)$ \\
\hline \multicolumn{6}{|c|}{ Non-vascular mortality: } \\
\hline No strong PPI & 17425 & 367 & 461 & - & - \\
\hline Strong PPI & 12012 & 299 & 541 & 1.72 (1.52 to 1.95$)$ & $1.71(1.51$ to 1.94$)$ \\
\hline
\end{tabular}

*Omeprazole, esomeprazole, lansoprazole.

†Captures whether patients were ever counted in this exposure group during follow-up.

$\ddagger$ Adjusted for age, sex, BMI, smoking, alcohol, diabetes, peripheral vascular disease, coronary heart disease, ischaemic stroke, cancer. 
Table 4| Association between ranitidine exposure and vascular/mortality outcomes in patients taking clopidogrel and aspirin

\begin{tabular}{|c|c|c|c|c|c|}
\hline \multirow[b]{2}{*}{ Exposure* } & \multirow[b]{2}{*}{ No of patients } & \multirow[b]{2}{*}{ Median follow-up(days) } & \multirow{2}{*}{$\begin{array}{l}\text { No of events (all cause mortality or } \\
\text { incident myocardial infarction) }\end{array}$} & \multicolumn{2}{|c|}{ Hazard ratio $(95 \% \mathrm{Cl})$} \\
\hline & & & & Crude & \\
\hline
\end{tabular}

Primary outcome

All cause mortality or incident myocardial infarction:

\begin{tabular}{|c|c|c|c|c|c|}
\hline No ranitidine & 23996 & 430 & 2655 & - & - \\
\hline Ranitidine & 1561 & 188 & 105 & $1.23(1.01$ to 1.50$)$ & $1.20(0.99$ to 1.46$)$ \\
\hline \multicolumn{6}{|c|}{ Secondary outcomes } \\
\hline \multicolumn{6}{|c|}{ All cause mortality: } \\
\hline No ranitidine & 24004 & 441 & 2140 & - & - \\
\hline Ranitidine & 1595 & 189 & 88 & 1.28 (1.04 to 1.59$)$ & 1.25 (1.01 to 1.55$)$ \\
\hline \multicolumn{6}{|c|}{ Incident myocardial infarction: } \\
\hline No ranitidine & 23996 & 430 & 709 & - & - \\
\hline Ranitidine & 1561 & 188 & 25 & 1.11 (0.74 to 1.65$)$ & 1.07 (0.72 to 1.59$)$ \\
\hline \multicolumn{6}{|c|}{ Vascular mortality: } \\
\hline No ranitidine & 24004 & 441 & 1185 & - & - \\
\hline Ranitidine & 1595 & 189 & 41 & 1.08 (0.79 to 1.47$)$ & $1.06(0.78$ to 1.45$)$ \\
\hline \multicolumn{6}{|c|}{ Non-vascular mortality: } \\
\hline No ranitidine & 24004 & 441 & 955 & - & - \\
\hline Ranitidine & 1595 & 189 & 47 & 1.54 (1.15 to 2.07 ) & $1.49(1.11$ to 1.99$)$ \\
\hline
\end{tabular}

${ }^{*}$ Captures whether patients were ever counted in this exposure group during follow-up.

†Adjusted for age, sex, BMI, smoking, alcohol, diabetes, peripheral vascular disease, coronary heart disease, ischaemic stroke, cancer. 
Table 5| Association between paroxetine/fluoxetine exposure and vascular/mortality outcomes in patients taking clopidogrel and aspirin

\begin{tabular}{|c|c|c|c|c|c|}
\hline \multirow[b]{2}{*}{ Exposure* } & \multirow[b]{2}{*}{ No of patients } & \multirow[b]{2}{*}{ Median follow-up (days) } & \multirow{2}{*}{$\begin{array}{l}\text { No of events (all cause mortality } \\
\text { or incident myocardial infarction) }\end{array}$} & \multicolumn{2}{|c|}{ Hazard ratio $(95 \% \mathrm{Cl})$} \\
\hline & & & & Crude & Fully adjusted $\dagger$ \\
\hline
\end{tabular}

Primary outcome

All cause mortality or incident myocardial infarction:

\begin{tabular}{|c|c|c|c|c|c|}
\hline No paroxetine/fluoxetine & 24004 & 429 & 2651 & - & - \\
\hline Paroxetine/fluoxetine & 1143 & 235 & 109 & $1.54(1.27$ to 1.87$)$ & 1.42 (1.17 to 1.72$)$ \\
\hline \multicolumn{6}{|l|}{ Secondary outcomes } \\
\hline \multicolumn{6}{|l|}{ All cause mortality: } \\
\hline No paroxetine/fluoxetine & 24049 & 440 & 2137 & - & - \\
\hline Paroxetine/fluoxetine & 1155 & 236 & 91 & $1.62(1.32$ to 2.00$)$ & 1.49 (1.20 to 1.84$)$ \\
\hline \multicolumn{6}{|c|}{ Incident myocardial infarction: } \\
\hline No paroxetine/fluoxetine & 24004 & 429 & 710 & - & - \\
\hline Paroxetine/fluoxetine & 1143 & 235 & 24 & $1.20(0.80$ to 1.81$)$ & $1.13(0.75$ to 1.69$)$ \\
\hline \multicolumn{6}{|l|}{ Vascular mortality: } \\
\hline No paroxetine/fluoxetine & 24049 & 440 & 1183 & - & - \\
\hline Paroxetine/fluoxetine & 1155 & 236 & 43 & 1.38 (1.02 to 1.88$)$ & 1.29 (0.95 to 1.75$)$ \\
\hline \multicolumn{6}{|l|}{ Non-vascular mortality: } \\
\hline No paroxetine/fluoxetine & 24049 & 440 & 954 & - & - \\
\hline Paroxetine/fluoxetine & 1155 & 236 & 48 & 1.93 (1.44 to 2.58$)$ & $1.73(1.29$ to 2.31$)$ \\
\hline
\end{tabular}

${ }^{*}$ Captures whether patients were ever counted in this exposure group during follow-up.

†Adjusted for age, sex, BMI, smoking, alcohol, diabetes, peripheral vascular disease, coronary heart disease, ischaemic stroke, cancer. 
Table 6| Association between citalopram exposure and vascular/mortality outcomes in patients taking clopidogrel and aspirin

\begin{tabular}{|c|c|c|c|c|}
\hline & & & No of events (all cause mortality or & Hazard ratio $(95 \% \mathrm{Cl})$ \\
\hline Exposure $^{*}$ & No of patients & Median follow-up (days) & incident myocardial infarction) & Fully adjusted $\dagger$ \\
\hline
\end{tabular}

Primary outcome

All cause mortality or incident myocardial infarction:

\begin{tabular}{|c|c|c|c|c|c|}
\hline No citalopram & 23888 & 421 & 2553 & - & - \\
\hline Citalopram & 1930 & 225 & 207 & $1.63(1.42$ to 1.88$)$ & 1.52 (1.32 to 1.76$)$ \\
\hline \multicolumn{6}{|c|}{ Secondary outcomes } \\
\hline \multicolumn{6}{|c|}{ All cause mortality: } \\
\hline No citalopram & 23893 & 432 & 2049 & - & - \\
\hline Citalopram & 1960 & 229 & 179 & 1.74 (1.49 to 2.02$)$ & 1.61 (1.38 to 1.88$)$ \\
\hline \multicolumn{6}{|c|}{ Incident myocardial infarction: } \\
\hline No citalopram & 23888 & 421 & 690 & - & - \\
\hline Citalopram & 1930 & 225 & 44 & $1.26(0.93$ to 1.71$)$ & 1.14 (0.84 to 1.56$)$ \\
\hline \multicolumn{6}{|c|}{ Vascular mortality: } \\
\hline No citalopram & 23893 & 432 & 1137 & - & - \\
\hline Citalopram & 1960 & 229 & 89 & 1.54 (1.24 to 1.92$)$ & 1.44 (1.16 to 1.79$)$ \\
\hline \multicolumn{6}{|c|}{ Non-vascular mortality: } \\
\hline No citalopram & 23893 & 432 & 912 & - & - \\
\hline Citalopram & 1960 & 229 & 90 & 1.98 (1.59 to 2.46$)$ & 1.84 (1.48 to 2.29$)$ \\
\hline
\end{tabular}

${ }^{*}$ Captures whether patients were ever counted in this exposure group during follow-up.

†Adjusted for age, sex, BMI, smoking, alcohol, diabetes, peripheral vascular disease, coronary heart disease, ischaemic stroke, cancer. 
Table 7| Self controlled case series analysis. Association between exposure to proton pump inhibitor (PPI), ranitidine, paroxetine/fluoxetine, or citalopram and incident myocardial infarction (defined by MINAP (Myocardial Ischaemia National Audit Project)) ${ }^{\star}$ in patients taking clopidogrel and aspirin

\begin{tabular}{|c|c|c|c|c|}
\hline Exposure & No of patientst & Patient years & No of eventsł & Age adjusted IRR $(95 \% \mathrm{Cl})$ \\
\hline No PPI & 430 & 708 & 375 & - \\
\hline PPI & 444 & 719 & 395 & $0.75(0.55$ to 1.01$)$ \\
\hline 14 days before PPI & 201 & 8 & 10 & $1.39(0.71$ to 2.70$)$ \\
\hline No strong PPI & 478 & 749 & 398 & - \\
\hline Strong PPI & 432 & 677 & 372 & 0.77 (0.57 to 1.03$)$ \\
\hline 14 days before strong PPI & 208 & 8 & 10 & 1.34 (0.69 to 2.59$)$ \\
\hline No ranitidine & 641 & 1373 & 743 & - \\
\hline Ranitidine & 76 & 52 & 26 & 0.57 (0.31 to 1.06$)$ \\
\hline 14 days before ranitidine & 48 & 2 & 2 & 1.10 (0.26 to 4.65$)$ \\
\hline No paroxetine/fluoxetine & 603 & 1384 & 735 & - \\
\hline Paroxetine/fluoxetine & 44 & 47 & 31 & $1.65(0.87$ to 3.15$)$ \\
\hline 14 days before paroxetine/fluoxetine & 26 & 1 & 1 & $1.35(0.17$ to 10.62$)$ \\
\hline No citalopram & 632 & 1343 & 727 & - \\
\hline Citalopram & 79 & 78 & 43 & $0.84(0.49$ to 1.45$)$ \\
\hline 14 days before citalopram & 59 & 2 & 0 & - \\
\hline
\end{tabular}

IRR=incident rate ratio.

*Analysis includes patients not exposed to PPIs to improve age adjustments.

†Captures number of patients ever included in each exposure group.

łlncludes repeat events where observed during study period. 


\section{Figures}



Fig 1 Pictorial representation of study design; indicative single patient timeline

\section{Exposure}

Proton pump inhibitor

Cohort:

All cause mortality and myocardial infarction All cause mortality Myocardial infarction

Vascular mortality

Non-vascular mortality

Self controlled case series-myocardial infarction

\section{Ranitidine}

Cohort:

All cause mortality and myocardial infarction All cause mortality Myocardial infarction

Vascular mortality

Non-vascular mortality

Self controlled case series-myocardial infarction

\section{Paroxetine/fluoxetine}

\section{Cohort:}

All cause mortality and myocardial infarction All cause mortality Myocardial infarction

Vascular mortality

Non-vascular mortality

Self controlled case series-myocardial infarction

\section{Citalopram}

Cohort:

All cause mortality and myocardial infarction All cause mortality Myocardial infarction Vascular mortality Non-vascular mortality

Self controlled case series-myocardial infarction
Relative risk $(95 \% \mathrm{Cl})$

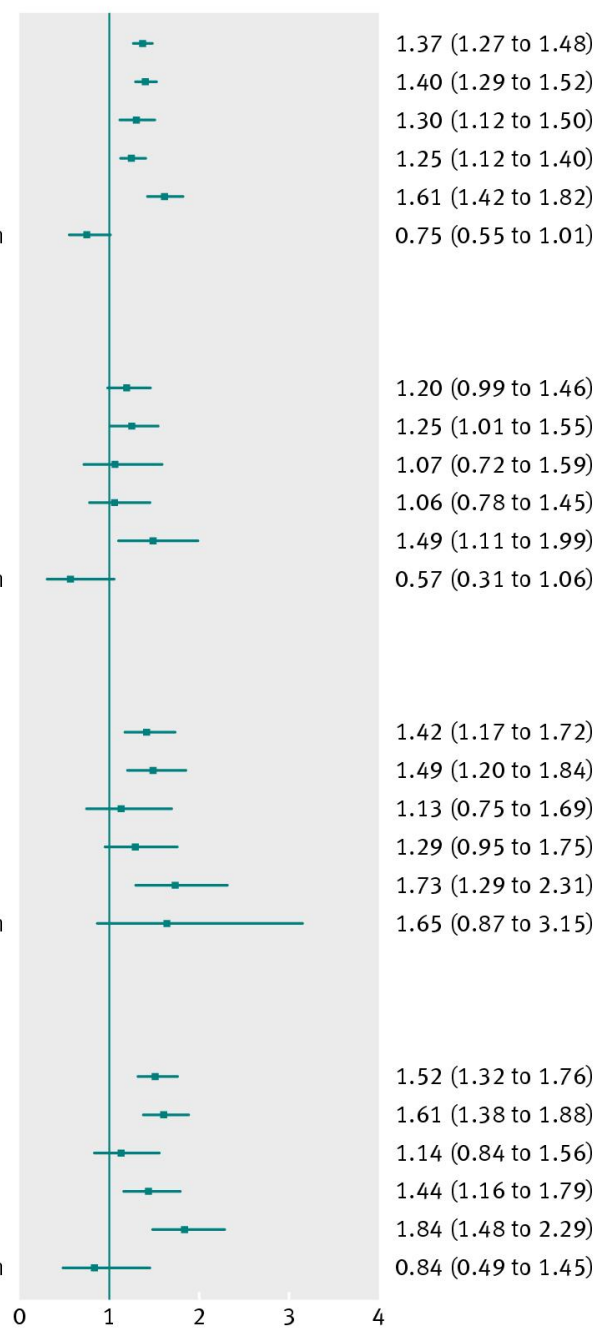

Fig 2 Association between proton pump inhibitors, ranitidine, paroxetine/fluoxetine, or citalopram and all outcomes in cohort analysis and self controlled case series. Relative risk expressed as hazard ratio for cohort analysis and incidence rate ratio for self controlled case series 